\title{
A case-study of the low-latitude thermosphere during geomagnetic storms and its new representation by improved MSIS model
}

\author{
T. K. Pant, R. Sridharan \\ Physical Research Laboratory,Navrangpura, Ahmedabad 380 009, India Fax: +796560502; e-mail: tarun@prl.ernet.in
}

Received: 12 May 1997 / Revised: 27 February 1998 / Accepted: 2 March 1998

\begin{abstract}
The thermospheric temperatures from low and equatorial latitudes during geomagnetically disturbed periods are known to exhibit significant deviations from atmospheric model predictions. Also, the oscillatory features seen in the observations are not accounted for by the models. A simple relation has been established between the difference in the observed and modelpredicted temperatures and the rate of change of Dst, the magnetic index representing the ring current variabilities. Using this relation, a correction term has been added to the latest MSIS-90 model algorithm and almost all the observed variations in neutral temperatures spectroscopically determined from Mt.Abu, a lowlatitude station in India, are successfully reproduced for two moderate geomagnetic storms.
\end{abstract}

Key words. Low-latitude thermosphere - MSIS model · Stormtime model predictions, FP spectroscopic temperatures

\section{Introduction}

The behaviour of the low-and equatorial-latitude thermosphere ionosphere system during geomagnetic storms and its representation by atmospheric models is a topic of active research. It is now accepted that the different atmospheric regions exist as a closely coupled system. Detailed investigations using data from multiple techniques and coordinated observations along with modelling are needed to unravel the complexities of the interactive processes and hence the coupling of the different atmospheric regions. One of the least explored aspects is the high-latitude-low-latitude coupling, i.e. how the large amount of energy that gets dumped into

Correspondence to: T. K. Pant the high-latitude regions, mostly in the form of particle precipitation, gets redistributed to other low/equatorial regions. This aspect becomes important during geomagnetically disturbed periods when the energy input enhances manifold and is crucial in the understanding of this magnetosphere-ionosphere-thermosphere phenomena. Strong meridional winds and gravity waves follow a series of highly energetic processes that get initiated at high latitude and are believed to play important roles in the redistribution of energy and momentum (Prolss, 1982). Also, it is known that there are several localized processes during a geomagnetic storm due to which neutral composition as well as ionospheric densities undergo significant changes (Jacchia, 1959; Taeusch et al., 1971; Trinks et al., 1975; Prolss, 1982; Mayr et al., 1978). Under these circumstances, a meridional circulation cell gets developed with equatorward flow in the upper and return flow in the lower thermosphere. As a consequence, at middle and low latitudes, the atmosphere undergoes an adiabatic compression causing a rise in the thermospheric temperatures (Armstrong, 1969; Hays and Roble, 1971; Rishbeth, et al., 1975). Further, the energy injection at high latitudes triggers a broad spectrum of gravity waves which in turn dissipate their energy by heat conduction, viscosity and ion drag during their propagation (Richmond, 1978; Hunsucker, 1982). The temperature at low latitudes rises and hence manifests itself as a geomagnetic effect (Klostermeyer, 1973; Mayr and Volland, 1974; Richmond, 1979; Prolss, 1982; Forbes et al., 1987; Burns and Killeen, 1992). In addition to this, the mapping of the magnetospheric convection electric fields to lower altitudes results in the enhancement of auroral electrojet currents. The consequent joule heating is an additional major source of heat. Apart from this, the hydromagnetic waves, getting generated at the bow shock, are also considered as a potential source. Though the magnitudes of the storm effects in the lower thermosphere are smaller at low latitudes, the effects do persist for a significantly longer periods even after the storm subsides (Fuller-Rowell and Rees, 1981). Quite a 
few temperature measurements from low and equatorial regions revealed enhancements to the tune of $100-400 \mathrm{~K}$ over the equator (Taeusch et al., 1971; Hernandez et al., 1975; Biondi and Meriwether, 1985; Prolss and Roemer, 1987), the magnitude being highly variable depending upon the intensity of the storm, its time of occurrence, duration, season and also on the location of the observing site. It should be mentioned here that though in general the alterations over low latitudes are caused by the high-latitude processes, there are instances wherein evidence has been obtained for direct deposition of energy overhead as well (Gupta et al., 1986). The energetic particles originating from the ring current are believed to play a crucial role in this region (Tinsley, 1981). Therefore one of the important aspects of the study of the atmospheric system during a geomagnetic storm is to be able to investigate its response for a sudden surge of energy input as compared to the gradually changing solar activity or seasonal effects. Individual case-studies are extremely relevant in this regard. These studies would eventually lead us to quantify and account for certain energetic processes into a global atmospheric model, thus making it applicable to a wide variety of geophysical conditions. Even the most comprehensive atmospheric model available to date is seen to have severe limitations in the representation of low and equatorial latitudes (Gurubaran and Sridharan, 1993, 1995). Gurubaran and Sridharan (1995), while compiling the thermospheric temperature data from Mt. Abu, one of the low-latitude stations in the Indian zone [right over the crest region of the equatorial ionization anomaly (EIA)], observed that the MSIS model represents the observational latitudes pretty well for low solar activity conditions and for geomagnetically quiet periods. However, as the solar activity picks up, the deviations between the measured and the model temperatures start increasing. Most of the times the model underestimates the temperatures. It was suggested by them that the model probably does not take into account all the essential energy inputs which incidentally become significant with the increase in solar activity. These observed deviations are not only seen in the absolute magnitudes but also in their temporal variabilities. On several occasions large-scale wave structures with periodicities ranging from $30-40 \mathrm{~min}$ are observed to $3 \mathrm{~h}$ which are not predicted by the model for the prevailing geophysical conditions. In the light of these facts, a case-study has been performed on two moderate geomagnetic storms during 1993-1994, a low solar activity period, making use of the spectroscopically obtained thermospheric temperature from Mt.Abu and those estimated from MSIS-90 model (Hedin, 1991) for the period of our observations. An appropriate additional factor to the model has been worked out by parameterizing one of the geomagnetic indices, Dst, and it has been demonstrated conclusively that with the suggested additive term the MSIS model could be made to reproduce all the observed periodicities including a negative swing with respect to the model values. The unaccounted differences, mostly restricted to premidnight hours on several occasions, are suggested to be associated with the geophysical processes peculiar to the equatorial and low latitudes. These aspects are presented and discussed below.

\section{Experimental details}

A 100-mm, clear-aperture, pressure-scanned, FabryPerot spectrometer is being operated routinely from the Optical Aeronomy Laboratory at Mt. Abu $\left(24.6^{\circ} \mathrm{N}\right.$, $72.7^{\circ} \mathrm{E}, 20.3^{\circ} \mathrm{N}$ dip lat.) during favourable viewing conditions. The central aperture scanning is accomplished by means of a programmable piston drive. The OI 630.0-nm emission line originating from the thermosphere is selected and isolated by means of a temperature-tuned narrow-band (FWHM-3 $\mathrm{A}^{\circ}$ ) interference filter. A photomultiplier-based photon counting system is activated by means of the piston drive. The pressure and photon counts are synchronously detected by means of a PC-based data acquisition system. The instrument is operated at a finesse of $\sim 8$, the instrumental broadening being estimated using a $\mathrm{He}-\mathrm{Ne}$ laser. The $F P$ chamber is precisely temperature controlled to $\sim 0.1{ }^{\circ} \mathrm{C}$ in order to determine the Doppler shifts as well. Further details of the experiment and the data reduction procedure are available in the literature (Gurubaran and Sridharan, 1993).

\section{Data presentation}

Two moderate geomagnetically disturbed events, one during 6-10 December 1993 and another during 5-14 February 1994, have been chosen for the present study. It is understood that these two events, though they at the outset may appear as moderate storms, are indeed very special events. The $B_{z}$ component of the Interplanetary Magnetic Field (IMF) remained southward for several hours, a rare phenomenon, and the polar geophysical observatory Eureka $\left(80^{\circ} \mathrm{N}, 90^{\circ} \mathrm{W}\right)$ operated by the University of Saskatchewan, recorded a series of F-region patches lasting for several hours and moving across the poles (Don J. McEwen, personal communication). Figure 1 depicts the $D s t$ values for both the events; part 1a shows a classical storm sequence with the main phase of the storm during 7-8 December. The recovery phase starts during 8-9 extending up to 10-11 December, beyond which a slow recovery is seen up to 15 December. Figure $1 \mathrm{~b}$ depicts the second event, the intensity of which is nearly double that of the previous one. Very large scale oscillatory features are seen for the whole period from 5 to 14 February. The recovery on this occasion is rather slow and it could well represent a recurring storm, in which case it would represent a complex dynamic situation. Figure 2 depicts the measured neutral temperatures $T_{n}$ by dots with error bars along with the MSIS-90 model values predicted for this occasion (dashed line). On top of the panel the $A p$ values are also provided. On 8 December when the $A p$ value is $\sim 47$ the measured $T_{n}$ showed large deviations from the model values during the pre-midnight hours. 

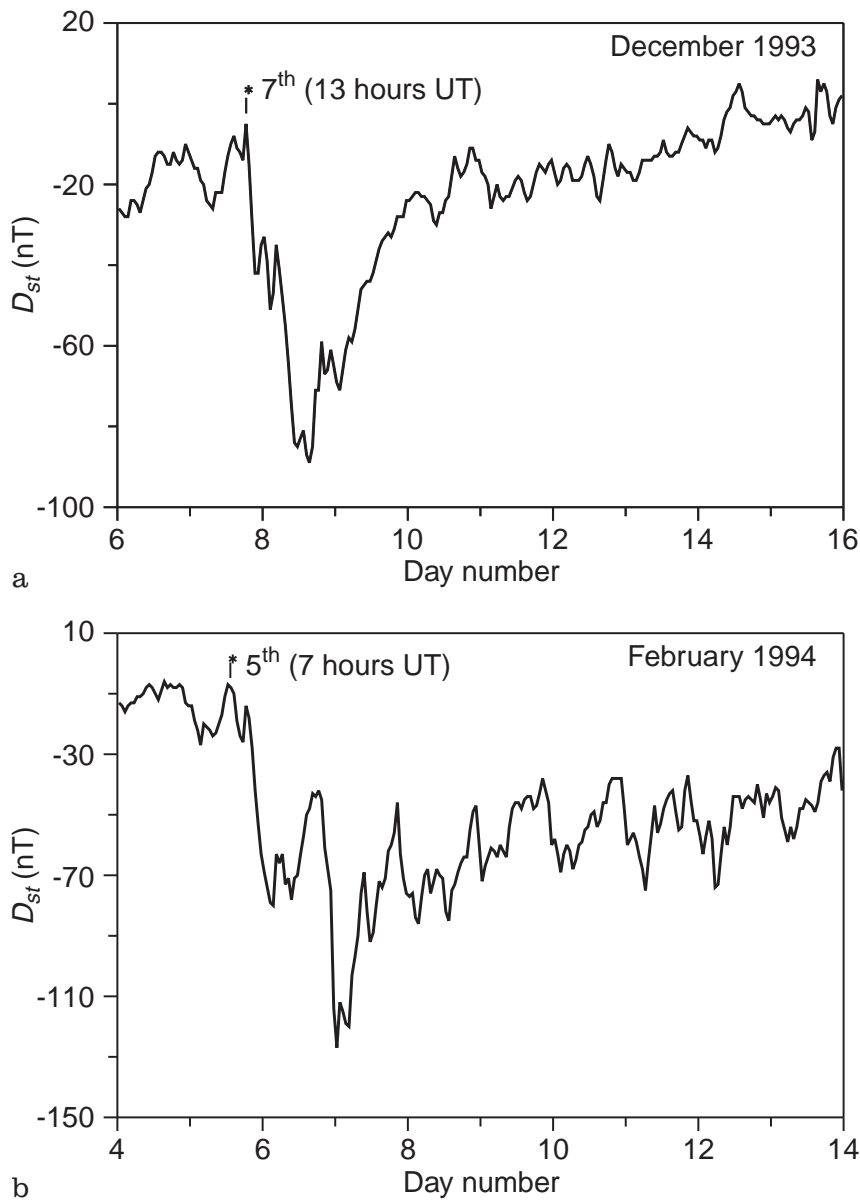

Fig. 1a,b. Variation of Dst index for the periods under study during a December 1993, b February 1994

While the model predicted montotonically decreasing temperatures from $\sim 850$ to $800 \mathrm{~K}$ over an 8 -h timeperiod (dashed line), the measurements showed an initial decrease and shot up to as high as $1500 \mathrm{~K}$ around 2200 IST. There had been a gap in the data due to decreased airglow intensities up to 0100 IST. During that timeinterval the temperatures had come down to $\sim 900 \mathrm{~K}$ and continued to be $\sim 800 \mathrm{~K}$ for the rest of the period. The very next day, i.e. 9 December, the $A p$ value had gone down to as low as 3 . The neutral temperatures too showed values ranging from $800-1100 \mathrm{~K}$ but it showed an oscillatory feature with a periodicity of $\sim 2 \mathrm{~h}$. The MSIS-90 model value for this day once again predicted a nearly flat, featureless temperature profile with a $T_{n}$ of $\sim 800 \mathrm{~K}$. The second event on 5 February, when the $A p$ value was at 33 , showed large-scale oscillatory features in temperature with $\sim 2 \mathrm{~h}$ periods and with a peak value $\sim 1100 \mathrm{~K}$ while the MSIS model once again predicted a flat $T_{n}$ profile of $\sim 800 \mathrm{~K}$. The data for 6 February revealed still larger temperature fluctuations, the peak value reaching $1300 \mathrm{~K}$ and larger periods of $\sim 3 \mathrm{~h}$. The $A p$ value on this day was 61 and the MSIS model did not envisage any change in the temporal variation of $T_{n}$.
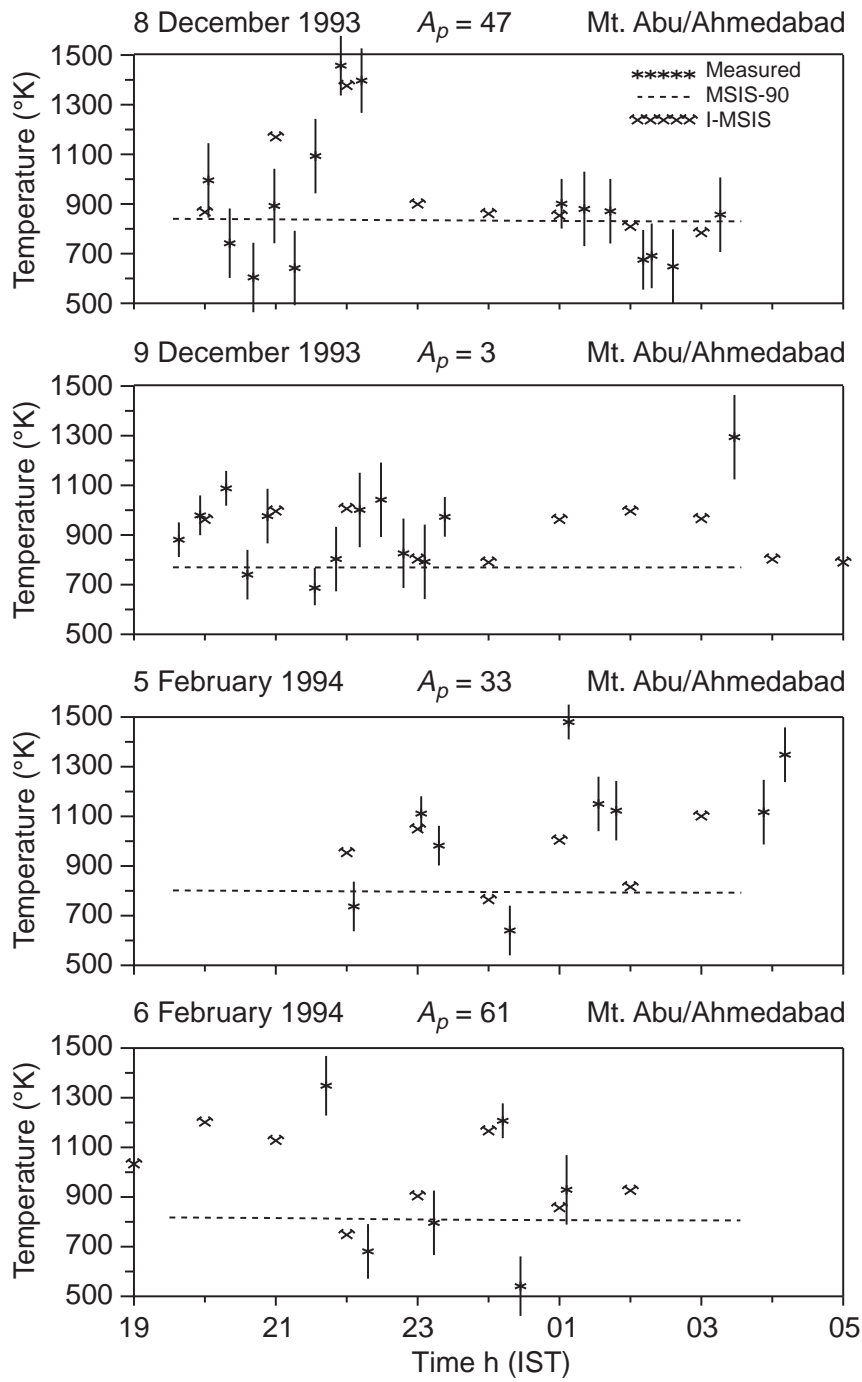

Fig. 2. Variation of spectroscopically measured thermospheric temperatures from Mt.Abu (dots with error bars) along with MSIS90 model prediction (dashed line) for different days under consideration (the date and the $A p$ values are shown on top of the panel). It can be seen that the improved MSIS values (crosses) agree well with the measured temperatures.

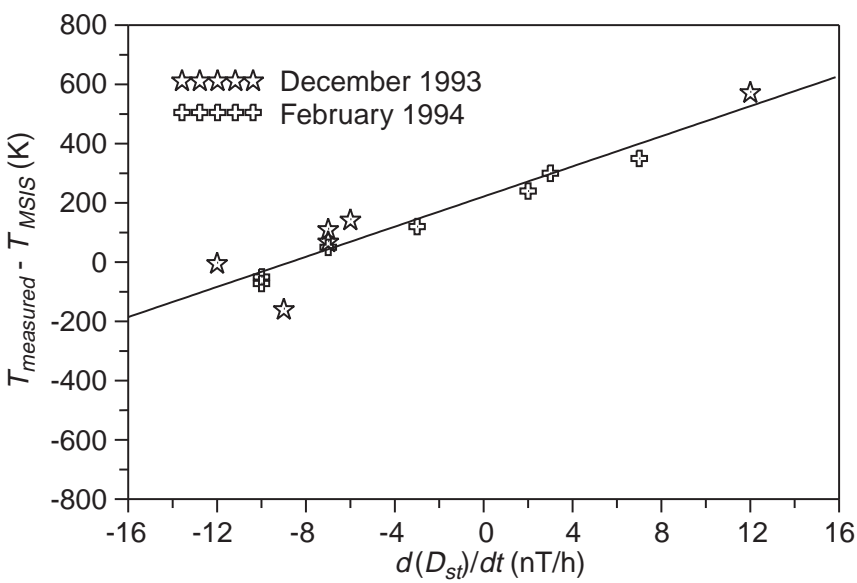

Fig. 3. Scatter plot considering temperatures on 8 December 1993 and 5 February 1994 


\section{Improvement of the MSIS model}

In order to increase the utility of an atmospheric model, it becomes important to augment the same by appropriately incorporating certain unaccounted factors after suitably parameterizing them. Since a large quantity of data from low latitudes consistently reveal that the model tends to underestimate the neutral temperatures, more so in disturbed periods, certain additional parameters need to be incorporated in the model. Till now the prediction of thermospheric variations and perturbations in empirical models are keyed to the geophysical indices like $K p$ and $A p$ which are taken to represent the geophysical processes to a good measure. However, these indices might not be representing the actual conditions especially over low latitudes (Almar et al., 1996) and they tend to smooth out the variations. Making the model solely depend on the $K p$ and $A p$ indices would have limitations, and these will be discussed later. Instead of $K p$ and $A p$ indices the $D s t^{\prime}$, another storm time index might serve to represent the equatorial and low latitudes better (Almar et al., 1992) as this best approximates a well-defined phenomenon, namely the ring current. It is a measure of the strength of symmetric ring current during storms over the equatorial region. In the present study an attempt has been made to parameterize Dst in order to be able to explain the observed thermospheric temperatures. The logic that has been adopted is as follows: during the main phase of any geomagnetic storm, energy is pumped into the ring current and it builds up. Later, in the recovery phase, the stored energy gets released into the system more favourably at high latitudes. As mentioned earlier, advection, diffusion and travelling atmospheric disturbances redistribute the energy in the form of gravity waves. There would be a time-delay before the low-latitude region would start experiencing the impact of the geomagnetic storm. The rate at which the Dst parameter changed with time, i.e. $\mathrm{d}(D s t) / \mathrm{d} t$, was taken as a measure of the energy input into the atmospheric system. The rationale for treating it so is discussed later. By considering both the storms a detailed correlation analysis was performed and a high correlation of $\sim 0.8-0.9$ was seen between the $\mathrm{d}(D s t) / \mathrm{d} t$ and the $T_{n}$ variations when the former is delayed by 16-18 h (Fig. $3)$. This time-delay is considered to be reasonable in compliance with the available information on meridional winds and gravity-wave propagation characteristics. For the December data a relation was established between the deviations in the measured and model predicted temperatures $\left(\Delta T_{n}\right)$ and the rate at which the $D s t$ variations occurred before $18 \mathrm{~h}$. A simple empirical relationship was arrived at

$\Delta T_{n}=19.124 \times \frac{\mathrm{d}(D s t)}{\mathrm{d} t}+171.464$.

The same relation was arrived at for the February 1994 data when a similar excercise (as for December 1993) was carried out independently. This sort of relation could therefore be considered representative of this particular solar epoch, the constant additional term would then represent the contribution from other potential agencies. The presence of the constant term in the empirical relation clearly indicates that all the sources accounted for by the model are insufficient. In the absence of the exact quantitative estimates of contribution from other potential sources, e.g lowlatitude processes, the only statment which can be made is that this constant term represents the background thermospheric condition as it is under the influence of these local processes almost all the time. This aspect and the limitations imposed by these local processes are discussed in the text to follow. Using the foregoing relation to represent the periods under consideration, the $\Delta T_{n}$ values were estimated for the remaining three nights. This $\Delta T_{n}$ is treated as an additive term to the standard MSIS model temperature. The estimated temperatures using this empirical relationship (after applying the correction to the MSIS model) are depicted in Fig. 2 by crosses, while the actual measurements are represented by dots. One can notice that the observed features are now reproduced remarkably by the improved MSIS (I-MSIS) model. The large shooting up of temperature at $\sim 2200$ IST on 8 December 1993, the oscillatory features of 5 and 6 February 1994, do get reproduced fairly well. Temperature data of 9 December revealed much faster fluctuations, with periodicities of $<1 \mathrm{~h}$. Since the Dst data used in the present analysis are from the hourly $D s t$ values, smaller periods could not be extracted. At least 10-min Dst values would be needed for a further detailed study. In spite of this limitation the I-MSIS model values are seen to pass through the actual data points.

\section{Discussion}

The limitations in the present-day models in representing the atmosphere in general, and the low and equatorial latitudes in particular, are well known, and the fact that these are due to the complexities of the associated geophysical processes is also well appreciated. Currently neither empirical nor analytical models could represent the atmospheric system to the desired level and therefore continuous attempts are underway for their improvement. The thermosphere is a non-linear medium, one which gets strongly affected by changes in the external energy mainly from the sun, followed by the magnetosphere, the middle and lower atmospheres. While the processes related to the direct solar heating are straight forward, the ones related to the other sources are rather complicated, which make it difficult for any of the existing models to predict the behavioural pattern of the thermospheric parameters. As discussed earlier, present-day models, for e.g. MSIS, take the geomagnetic indices $K p$ as a measure of the energy input into the atmospheric system from the magnetosphere. This index, as the suffix indicates, is rather a reference with respect to the planetary level of disturbance and are used as the basic elements for the statistical studies of the time variations of geomagnetic activity, and of its 
relationship with other terrestrial variations. However, the $K p$ does not properly represent the periods and also the magnetic activity levels with sufficient time resolution to be considered a good reference for the classification of periods. The limitations in the usage of $K p$ as a reference are (1) lack of information on the universal time variation of magnetic activity, (2) the summer and winter hemisphere differences are summarily nullified, though such differences are known to be real, and (3) the standardization raises arbitrarily the $K p$ to a certain night level and it is not done in a homogeneous way (Mayaud, 1975).

The magnetic storm is understood to be middle- and low-latitude geomagetic variations identified by the intensification of the ring current and the source of low-frequency component of magnetic variation; it is represented by the $D s t$ index. This index is sensitive to variable magnetic fields whose source is remote and is distinctly different from the polar storm time variations. In this context, it had been suggested that Dst could be a better index to be incorporated in the atmospheric models (Almar et al., 1996). While explaining the total neutral density measurements obtained from 'CACTUS' satellite accelerometer data, Almar et al. (1992) showed that the $D s t$ index, instead of the $K p$, provides a much better agreement of the model with the measurements. Hernandez and Roble (1978) showed a linear relation between the $\mathrm{d}(D s t) / \mathrm{d} t$, the rate at which energy gets transferred into and from the earth's ring current and the meridional velocities over Fritz peak $\left(39^{\circ} \mathrm{N}, 105.5^{\circ} \mathrm{W}\right)$, a mid-latitude station. Quite a few similarities were shown to be prevalent in both the parameters. On certain occasions there had been a timedelay of a few hours. The first positive indication for a possible means of quantifying the rate of energy release $\mathrm{d}(D s t) /(\mathrm{d} t)$ as a useful parameter for global thermospheric 'dynamic' models was thus provided by them.

In the present paper, it is being demonstrated that the same parameter, if suitably accounted for, could also be useful in making the static models represent the atmospheric system reasonably well. The $16-18 \mathrm{~h}$ time-delay invoked in the choice of $\mathrm{d}(D s t) / \mathrm{d} t$ stands for the response time for the latitude region of $\mathrm{Mt}$. Abu $\left(24.6^{\circ} \mathrm{N}, 72.7^{\circ} \mathrm{E}, 20.3^{\circ}\right.$ dip lat.) to get influenced owing to the energy input at high latitudes. It would depend on the level of magnetic activity and also on the geophysical location of the observing station. The events under investigation in the present study were moderately disturbed periods and the solar activity too was rather low and the season represented winter months. Earlier studies of the response of the equatorial thermosphereionosphere system using DE-2 data revealed that in the morning sector during summer months, it takes about 12-h for the storm effects to be registered in the neutral temperatures (Burns and Killeen, 1992) and it was ascribed to gravity waves triggered by the storm. However, Richmond (1979) suggested that meridional circulation is also important and could take $\sim 10-12 \mathrm{~h}$. In the results discussed in the present paper, the preferred time-delay is significantly longer and it could be due to the steady transequatorial winds from the summer hemisphere opposing the equatorward flow in the winter hemisphere during the storm. By suitably incorporating the Dst parameter into the model, the temporal variabilities are seen to be fairly well predicted. Any deviation could be due to one or many of the aspects discussed below. The variations in Dst are not solely due to the ring currents because the low-latitude perturbation magnetic field is also influenced by contributions from substorm related current systems for, e.g., the asymmetric ring current involving field-aligned currents (Fukushima and Kamide, 1972), the ionospheric currents (Takahashi et al., 1991) and also by the occasional collapse of the cross-tail current near the inner edge of the plasma sheet. It is very difficult to separate out the contributions of these elements from the integrated magnetic field variations in order to account for the symmetric ring currents alone. The inherent difficulties in using the Dst index straight away still need to be quantified by systematic studies. In addition to this, certain other factors might also affect the spectroscopic measurements. It is well known that the centroid of emission of $O I$ 630.0-nm airglow lies $\sim 250 \mathrm{~km}$ and the semithickness of the emission layer is $\sim 70 \mathrm{~km}$. This region of emission lies in the height region where the temperature profile shows a steep gradient. This would cause a distortion in the emission profile which would manifest as larger temperatures. Simulation studies for typical conditions have revealed that it could be anywhere between 5 and $10 \%$. Further, the region of present study, Mt.Abu happens to be located under the crest of the Equatorial Ionisation Anomaly (EIA). On several occasions the EIA crest even crosses beyond Mt.Abu only to return back equatorwards during night-time. This would result in steep spatial gradients in the airglow intensity (Sridharan et al., 1993) which might in turn distort the profile. In addition to the preceding, unique geophysical phenomena like the Equatorial Temperature and Wind Anomaly (ETWA), which essentially deals with the coupling of the neutral atmosphere and the $F$ region of the ionosphere through ion-drag (Raghavarao et al., 1991) and chemical heating (Fuller-Rowell et al., 1997), the Midnight Temperature Maximum (MTM) (Spencer et al., 1979), the Equatorial spread-F associated heating of the neutral atmosphere (Cole, 1974; Rajaraman et al., 1979), etc. act as highly varying sources of energy, more so during magnetically quiet periods. It is believed that by parameterizing these processes and suitably incorporating them in the existing models, it would become possible to make the models represent the low-latitude regions even better during magnetically quite periods.

\section{Conclusion}

The present exercise, an attempt to study and account for the differences $\left(\Delta T_{n}\right)$ in the MSIS-90 model and spectroscopically measured neutral temperatures from a low-latitude station Mt.Abu, revealed that the temporal variations in the measured temperatures had a one-toone correlation with that in the rate of change of $D s t$ 
(magnetic index), 16-18 $\mathrm{h}$ beforehand, indicating that the thermospheric energy budget at these locations is significantly modulated by the high-latitude processes, bringing out the truly global nature of these effects. It has been interpreted that the transfer of energy and momentum from high to low latitudes takes about that much time during the given season and moderately disturbed low solar activity periods. A simple empirical relationship for the period and conditions under consideration has been obtained between the rate at which energy transfer takes place to and from the earth's ring current, $\mathrm{d}(D s t) / \mathrm{d} t$, and the deviations of the measured thermospheric temperature from the model $\left(\Delta T_{n}\right)$. The temperatures as predicted by the improved MSIS model (I-MSIS) brings out all the temporal variations of spectroscopically measured thermospheric temperatures.

Acknowledgements. Topical Editor D. Alcaydé thanks F. Barlier and another referee for the help in evaluating this paper.

\section{References}

Almar, I., E.Illes-Almar, A. Horvath, Z. Kollath, D. V. Bisikalo, and T. V. Kasimenko, Improvement of the MSIS 86 and DTM thermospheric models by investigating the geomagnetic effect, Adv. Space Res., 12, (6)313-(6)316, 1992.

Almar, I., E. Illes-Almar, A. Horvath, J. Kelemen, D. V. Bisikalo, T. V. Kasimenko, Investigation and modelling of an improved geomagnetic term for the CIRA'86 model at low latitudes, $A d v$. Space Res., 18, (9/10)375-(9/10)381, 1996.

Armstrong, E. B., Doppler shifts in the wavelength of the $O I \lambda 6300$ line in the night airglow, Planet. Space Sci., 17, 957-974, 1969.

Biondi, M. A., and J. W. Meriwether, Jr., Measured response of the equatorial thermospheric temperature to geomagnetic activity and solar flux changes, Geophys. Res. Lett., 12, 267-270, 1985.

Burns, A. G., and T. L. Killeen, The equatorial neutral thermospheric response to geomagnetic forcing, Geophys. Res. Lett., 19, 977-980, 1992.

Cole, K. D., Energetics of and a source of energy for equatorial spread $F$ events, J. Atmos. Terr. Phys., 36, 1099, 1974.

Forbes, J. M., R. G. Roble, and F. A. Marcos, Thermospheric dynamics during the March 22, 1979 magnetic storm, comparisons of model predictions with observations, J. Geophys. Res., 92, 6069-6081, 1987.

Fukushima, N., and Y. Kamide, Partial ring current models for worldwide geomagnetic disturbances, Rev. Geophys. Space Sci., 11, 795-853, 1972.

Fuller-Rowell, T. J., and D. Rees, Three-dimensional, time-dependent simulation of the global dynamical response of the thermosphere to a geomagnetic substorm, J. Atmos. Terr. Phys., 43, 701-721, 1981.

Fuller-Rowell, T. J., M. V. Codrescu, B. G. Fejer, W. Borer, F. Marcos, and D. N. Anderson, Dynamics of the low-latitude thermosphere: quiet and disturbed conditions, J. Atmos. Terr. Phys., 59, 1533-1540, 1997.

Gupta, R. J. N. Desai, R. Raghavarao, R. Sekar, R. Sridharan, and R. Narayanan, Excess heating over the equatorial latitudes during sudden commencement, Geophys. Res. Lett., 13, 10551058, 1986.

Gurubaran, S., and R. Sridharan, Effect of meridional winds and neutral temperatures on the $F$-layer heights over low latitudes, J. Geophys. Res., 98, 11629-11635, 1993.

Gurubaran, S., and R. Sridharan, Variabilities in the thermospheric temperatures in the region of the crest of the equatorial ionization anomaly, J. Atmos. Terr. Phys., 57, 695-703, 1995.

Hays, P. B, and R. G. Roble, Direct observations of thermospheric winds during geomagnetic storms, J. Geophys. Res., 76, 53165321, 1971.

Hedin, A. E., Extension of the MSIS thermosphere model into middle and lower atmosphere, J. Geophys. Res., 96, 1159-1172, 1991.

Hernandez, G., and R. G. Roble, Obervation of large-scale thermospheric waves during geomagnetic storms, J. Geophys. Res., 83, 5531-5538, 1978.

Hernandez, G., T. E. Vanzandt, V. L. Peterson, and J. P. Turtle, Comparisons of optical and incoherent-scatter measurements of nighttime exospheric temperatures at the magnetic equator, $J$. Geophys. Res., 80, 3271-3274, 1975.

Hunsucker, R. D., Atmospheric gravity waves generated in the high-latitude ionosphere. Rev. Geophys. Space Sci., 20, 293-315, 1982.

Jacchia, L. D., Two atmospheric effects in the orbital acceleration of artificial satellites, Nature, 183, 526, 1959.

Klostermeyer, J., Thermospheric heating by atmospheric gravity waves, J. Atmos. Terr. Phys., 35, 2267-2275, 1973.

Mayaud, P. N., Analysis of sudden storm commencements for the years 1868-1967, J. Geophys. Res., 80, 111-122, 1975.

Mayr, H. G., and H. Volland, Magnetic storm dynamics of thermosphere, J. Atmos. Terr. Phys., 36, 2025-2036, 1974.

Mayr, H. G., I. Harris, and N. W. Spencer, Some properties of upper atmosphere dynamics, Rev. Geophys. Space Phys., 16, 539-565, 1978.

Prolss, G. W., Perturbation of low-latitude upper atmosphere during magnetic substorm activity, J. Geophys. Res., 87, 52605266, 1982.

Prolss, G. W., and M. Roemer, Thermospheric storms, Adv. Space Res., 7, (10)223-(10)235, 1987.

Raghavarao, R., W. R. Hoegy, N. W. Spencer, and L. E. Wharton, An equatorial temperature and wind anomaly (ETWA), Geophys. Res. Lett., 18, 1193-1196, 1991.

Rajaraman T. N., J. N. Desai, and S. S. Degaonkar, Variation of $F$ region nighttime neutral temperatures with equatorial spread- $F$ activity, Proc. Indian Acad. Sci., (Earth and Plan. Sci.), 88, 69, 1979.

Richmond, A. D., Gravity wave generation, propagation and dissipation in the thermosphere, J. Geophys. Res., 83, 41314145, 1978.

Richmond, A. D., Thermospheric heating in magnetic storm: dynamic transport of energy from high to low latitudes, $J$. Geophys. Res., 84, 5259-5265, 1979.

Rishbeth, H., T. J. Fuller-Rowell, and A. S. Rodger, F-region storms and thermospheric circulation, J. Atmos. Terr. Phys., 37, 1055-1064, 1975.

Spencer, N. W., G. R. Carigan, H. G. Mayr, H. B. Niemann, R. F. Theis, and L. E. Wharton, The midnight temperature maximum in the earth's equatorial thermosphere, Geophys. Res. Lett., 6, 444-446, 1979.

Sridharan, R., R. Sekar, and S. Gurubaran, Two-dimensional highresolution imaging of the retrieval of the equatorial plasma fountain, J. Atmos. Terr. Phys., 55, 1661-1665, 1993.

Takahashi, S., M. Takeda, and Y. Yamada, Simulation of storm time partial ring current system and the dawn-dusk asymmetry of geomagnetic variation, Planet. Space Sci., 39, 821-832, 1991.

Taeusch, D. R., G. R. Carignan, and C. A. Reber, Neutral composition variation above $400 \mathrm{~km}$ during a magnetic storm, J. Geophys. Res., 76, 8318-8325, 1971.

Tinsley, B. A., Neutral atom prcipitation - a review, J. Atmos. Terr. Phys., 43, 617-632, 1981.

Trinks, H., K. H. Fricke, U. Laux, G. W. Prolss, and U. von Zahn, ESRO-4 Gas analyzer results: spatial and temporal structure of the midlatitude atmosphere during a geomagnetic storm, $J$. Geophys. Res., 80, 4571-4575, 1975. 